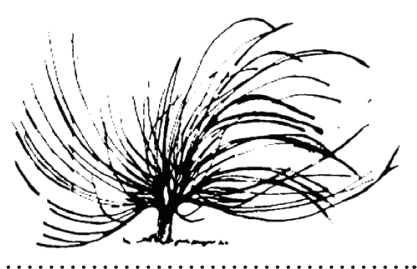

\title{
Introducción: la enseñanza de la Filosofía en la Universidad Nacional de Costa Rica (UNA)
}

En primera instancia, quisiéramos externarle nuestro profundo agradecimiento a Gabriela D'Odorico y Alejandro Cerletti por su tiempo, su disposición y sus valiosos aportes para discutir y complementar la formación filosófica-pedagógica que el profesor Juan Gómez, quien fue la chispa que encendió nuestro interés por la pedagogía crítica-transformadora, se esmeró en brindarnos en el transcurso de nuestros dos años como estudiantes activos del bachillerato en la Enseñanza de la Filosofía en el Centro de Investigación y Docencia en Educación (CIDE). Sin ustedes, nada de esto sería posible.

Es pertinente agradecer también a los académicos Jerry Murillo, director de Educología, Manuel Ortega, director de Filosofía y Sandra Ovares, vicedecana del CIDE, por su imprescindible ayuda en las gestiones administrativas, económicas y logísticas para generar y facilitar nuestras posibilidades de acción. Sin ustedes, nada de esto se hubiera desarrollado.

Ahora bien, ¿qué mejor forma de iniciar el presente libro que con una contextualización de su contenido?

Lo que ahora presentamos es el resultado de una serie de reflexiones y acciones que se concretizaron en un congreso denominado "Curso de aprovechamiento sobre la Enseñanza de la Filosofía: Indisciplinando 
la Didáctica". Las siguientes líneas discurrirán alrededor de las condiciones que posibilitaron la realización de dicho evento.

En la Universidad Nacional, el Bachillerato en Filosofía tiene un requisito que comparte solamente con el Bachillerato en Teología, y es la necesidad de aportar créditos complementarios de otra carrera. Siendo así, muchos de sus estudiantes encuentran varias limitaciones al respecto, lo que influye en la cantidad de graduados de ambas escuelas. Para paliar esta situación, y como respuesta a las solicitudes de un grupo de estudiantes que necesitaban soluciones al respecto, el entonces director de la Escuela de Filosofía, Manuel Ortega, asumió la tarea de gestionar, junto a la División de Educología, la posibilidad de que este grupo cursara el componente pedagógico correspondiente a la carrera de Enseñanza de la Filosofía, la cual llevaba aproximadamente diez años inactiva por falta de matrícula, en vez de tener que hacer todo el trámite de iniciar otra carrera y aportar los créditos correspondientes. La implicación sería, en estas circunstancias, que el título que recibiríamos en la graduación correspondería al de Enseñanza de la Filosofía, en vez de graduarnos en Filosofía con alguno de sus énfasis.

En vista de que la mayoría de las y los estudiantes que conformábamos ese grupo ya habíamos concluido (o estábamos a punto de concluir) el componente filosófico del plan de estudios, se brindó la posibilidad de llevar el pedagógico en forma de módulos; de manera que no tuviéramos que cursar de nuevo cuatro años para obtener el título de bachilleres. Iniciamos entonces ese caminar en el 2016, con el escepticismo y los prejuicios inherentes a la disciplina pedagógica en la contemporaneidad. Ese año correspondió al primer módulo pedagógico, en el que llevamos los cursos introductorios a la disciplina de manera regular; es decir, como estudiantes de primer ingreso en una nueva carrera.

El primer semestre de 2017 correspondió al segundo módulo. Ya para este año se modificó la modalidad de estudio: los módulos II y III eran intensivos y especializados, lo que implicaba una gran cantidad de tiempo tanto en términos presenciales de aula como de estudio independiente y un gran nivel de especificidad en la Enseñanza de la Filosofía. Cada módulo tenía un valor de catorce créditos, y correspondía a cuatro materias aproximadamente.

La lectura, discusión y problematización constante de libros y artículos fue parte fundamental en la dinámica implementada por el 
profesor Juan Gómez, además de sendas prácticas pedagógicas, giras interculturales, visitas a instituciones educativas de secundaria donde se impartiera la filosofía, así como el recurrir a la comunidad y a la familia para incursionar en saberes pedagógicos cotidianos. Entre la bibliografía correspondiente al segundo módulo se encontraban dos textos en los que Alejandro Cerletti participó como coautor y organizador: Didácticas de la filosofia: entre enseñar y aprender a filosofar y La enseñanza filosófica: cuestiones de política, género y educación; ambos de la editorial Noveduc.

Por motivo de una gira de Juan Gómez a la comunidad indígena Bribri de Kcha'bli (Kachabri en español) en Talamanca, como parte de las actividades del proyecto de Alfabetización crítica, se propuso suspender una de las sesiones presenciales del curso. Sin embargo, por la relevancia de los libros antes mencionados en nuestro contexto inmediato, la problematización de las temáticas didácticas y curriculares y la realización de la práctica profesional docente, hizo que la mayor parte del grupo decidiera reunirse para discutir e intercambiar ideas aun sin la presencia del docente. De esta "clase sin docente", o más bien, en la que fuimos docentes y estudiantes al mismo tiempo, surgió una idea que, en principio, era más un capricho que una posibilidad real: traer a Cerletti como ponente internacional. $Y$ es que nos impresionaba de sobremanera que en Sudamérica se estuviera problematizando y generando nuevo conocimiento sobre una disciplina que está, desde nuestra perspectiva, en peligro de extinción: la Enseñanza de la Filosofía.

La situación se dio, más o menos, de la siguiente manera: en vista de que hay autores que actualmente están produciendo respecto a esta temática, sería conveniente discutir de frente, con ellos, mientras sea posible. Teniendo en cuenta que la mayoría de los autores y autoras de los textos que consumimos ya fallecieron, esta era una posibilidad que no podíamos dejar tan fácilmente de lado. Teníamos entonces el capricho; lo que seguía era investigar las posibilidades de cómo realizarlo. Lo primero era averiguar si podíamos conseguir el presupuesto necesario para gestionar los gastos de transporte, estadía y alimentación; de manera que consultamos con la Escuela de Filosofía y la División de Educología. Jerry Murillo fue fundamental en todo este proceso, pues fue una de las personas que mayor ayuda nos brindó en general, no solo en lo económico, pues logró que la División de Educología nos financiara con un ponente, así como con la alimentación, también 
nos apoyó en lo administrativo, nos indicó los procedimientos que debíamos seguir para realizar las solicitudes correspondientes según la burocracia universitaria; procedimientos que, como era de esperar, no realizamos adecuadamente desde un inicio. Varias de las cartas que enviamos fueron rechazadas por falta de información, hasta que finalmente nos aprobaron, en Educología, el presupuesto requerido para traer a Alejandro.

Conseguimos el correo electrónico de Alejandro y le enviamos una invitación informal. Le explicamos superficialmente nuestra intención, y él, -fiel a su planteamiento de la aleatoriedad-, aceptó venir pese a la poca claridad, que existía aún en ese momento, sobre la actividad a realizar. Nos preguntó también si podía unirse su pareja, Gabriela D'Odorico, asumiendo ellos los gastos económicos correspondientes. De Gabriela no habíamos leído nada para ese momento, pero sabiendo el peso académico y la cantidad de aportes que podía realizar, -pues averiguamos que era experta en temáticas afines a lo que pretendíamos discutir, sobre todo en el campo del humanismo y la estética-, gestionamos también el presupuesto para traerla a ella en calidad de pasante, financiada el Decanato del CIDE.

El capricho preliminar empezaba a materializarse. Hay que mencionar también que todo este proceso se iba desarrollando en el transcurso del segundo semestre, lo que implicaba que la carga académica, aunada a la práctica profesional, a veces interferían en la posibilidad de responder adecuadamente a los plazos establecidos. Incluso nos olvidábamos de actualizar a Alejandro y a Gabriela sobre lo que estaba sucediendo, generándoles tanto expectativa como inseguridad, y con buena razón. En varias ocasiones el profesor Gómez tuvo que mediar entre ambas partes con el fin de comunicarle a los pasantes argentinos que la situación seguía en pie y que las y los estudiantes estábamos realizando las gestiones burocráticas del caso.

Seguía definir la naturaleza de la actividad. ¿Sería un congreso? ¿Una ponencia? ¿Mesa redonda? Luego de varias discusiones, decidimos que lo más adecuado sería plantear un curso de aprovechamiento, teniendo en cuenta que el profesorado de filosofía del Ministerio de Educación Pública (MEP) usualmente no tiene posibilidades de actualizarse en términos epistemológicos. Siendo así, se pensó en invitar a docentes del MEP y hacer de ellos y ellas el público meta. Debemos mencionar que, en este aspecto, la burocracia fue superior a nuestros 
esfuerzos: la asesoría de filosofía del Ministerio prácticamente se desentendió del asunto, y la jerarquía institucional se impuso.

Una vez planteada la actividad como un curso de aprovechamiento, debíamos precisar el cronograma correspondiente. Se propuso que, en las mañanas, la exposición fuera dirigida por Alejandro y Gabriela, y en las tardes las y los estudiantes presentaríamos ponencias en relación con temas pedagógicos. También el profesor Gómez aprovechó este espacio de la tarde para presentar sus aportes pedagógicos en forma de ensayo, mismos que están presentes en el escrito en cuestión.

Otra negociación fue la realizada por el profesor Gómez con la Revista Ensayos Pedagógicos. La revista aceptó dedicar un número especial para editar un libro que contuviera la producción de dicho Curso de Aprovechamiento de la Enseñanza de la Filosofía donde se discutiría sobre la necesidad de indisciplinar a la didáctica, pues Gómez considera que la pedagogía como tal es transdiciplinar, o cuando menos, interdisciplinaria, a pesar de las pretensiones cientificistas de muchos teóricos que la reducen a un saber instrumental, dejándola muy lejos de su carácter sociopráctico, crítico y transformador. En la Revista se ha manifestado total acuerdo con ese carácter crítico y revisionista de la pedagogía, como un área del saber que va más allá de la idea moderna de una disciplina que se explica a sí misma, que se debe a su objeto de estudio y que cumple una función preponderantemente instrumental.

Finalmente, el congreso se realizó del 6 al 10 de noviembre del 2017, contando con la participación del estudiantado del Módulo III de Enseñanza de la Filosofía, algunos docentes del MEP y estudiantes regulares de la carrera de Filosofía, además de la guía y el apoyo constante de Alejandro Cerletti, Gabriela D’Odorico y Juan Gómez. Como se mencionó anteriormente, los textos que a continuación se presentan son el resultado de la sistematización de las discusiones y las ponencias presentadas en ese evento. Se notará que los distintos escritos son propios de la pluma de siete estudiantes y tres profesores, en concordancia con el carácter indisciplinado del mismo congreso, pues parte de la horizontalidad del saber rompiendo con la clásica idea de que el conocimiento solo lo producen los expertos autorizados, esto es, quien posee el título que le acredite para ello.

El presente libro se dividirá en dos capítulos, y cada uno cuenta con cuatro artículos. El primer capítulo se titula Indisciplinando la 
didáctica de la enseñanza de la filosofia: acercamientos didácticos, antropológicos y estéticos.

Fiel al orden de lo desarrollado en el congreso, el libro inicia con un artículo escrito por Cerletti y D'Odorico. Ambos autores llaman la atención sobre varios problemas que atravesarán transversalmente en la presente obra.

En su artículo intitulado Indisciplinando la didáctica: reflexiones sobre una experiencia filosófico-pedagógica compartida destaca, en primer lugar, la necesidad de preguntarse por la posibilidad de una didáctica en términos filosóficos; es decir, la pregunta por las posibilidades de hablar de una metadidáctica que ponga en cuestión lo que implicaría abordar filosóficamente el asunto de aprender y enseñar.

En segundo lugar, la apreciación de que al hablar de un sujeto en educación este será entendido en términos de colectivo, tiene como consecuencia que podremos hablar de educación en tanto se procure que esta sea transformadora y emancipadora para aquellos y aquellas que participen del proceso de enseñanza-aprendizaje, en un sentido común.

En tercer lugar, se vislumbra un cuestionamiento a la razón instrumental y a la producción tecnológica, así como a sus respectivas aplicaciones en el ámbito educativo; ambas -exponen convencidosatraviesan, en este momento histórico, nuestra corporalidad y nuestra mente, y es labor de la filosofía interpelarlas -sin renunciar a ellas-, en vista de la amenaza latente que podría representar: la deshumanización de quienes son partícipes de los procesos educativos.

Finalmente, el problema del para qué filosofar -en conjunción con el para qué enseñar y aprender a filosofar- será una reflexión que se explicitará en los sentidos que le inspiran: lo aleatorio, bajo el marco del sentir, de la práctica de la igualdad; la recopilación de algunas de las experiencias de ambos, durante la realización del congreso, será el material que servirá para explorar estas cuestiones, bajo la lupa de la incertidumbre.

Por su parte, Daniel Porras y María José Romero, en su artículo El cine de terror en la enseñanza de la filosofía nos encaran a lo monstruoso, a la metáfora gráfica del cine -en el género del terror-, con la finalidad de hacernos evidente que pensar, crear, imaginar e interpretar son requisitos -dentro y fuera del salón de clases- para aprender y enseñar filosofía. 
Se trata de una propuesta didáctica guiada por el azar de la opinión del Otro, y por el acontecimiento (Badiou); nos muestran que es posible que nuestros miedos, la distopía, la búsqueda de lo extraño -el proceso de lo ajeno convirtiéndose en lo propio-, aquel temor en el que se inspira la necesidad de enviar miradas vacías al espejo, para distinguir lo foráneo -lo distinto-, es una excusa para enfrentarnos a lo real: somos nosotros quienes creamos nuestro mundo y lo hacemos por medio del análisis filosófico.

Sofía Cruz, Carlos Hernández y Luis Rodríguez nos invitan a desatar las cuerdas de nuestra garganta. Educación insumisa ante el pensamiento único: ¿quién te vigila? es un texto que pretende arrastrar elementos literarios de la obra 1984 de George Orwell a los contextos cotidianos educativos; se trata de un cotejo confrontativo de nuestra realidad con las pesadillas orwellianas sobre el control.

La vigilancia sistémica, la normalización, la violencia naturalizada, la resistencia, las posibilidades de transformación y emancipación, son protagonistas de esta revisión filosófica sobre nuestras realidades escolares. Los límites entre lo autómata y lo humano tratarán de ser dilucidados.

Los autores dejan en claro que la eliminación de lo aleatorio, de la incertidumbre, pone en duda la posibilidad de una educación que pretenda humanizar, y a su vez que sea posible plantear una didáctica que propicie las condiciones para el filosofar.

En el último artículo de este primer capítulo, escribe Óscar Rivas, desde el marxismo revisionista, nos presenta una relectura, contextualizada, del libro de Paulo Freire: Pedagogía del oprimido.

La primera generación de la Escuela Crítica de Frankfurt (Adorno, Horkheimer y Fromm), en conjunción con algunos referentes latinoamericanos (Hinkelammert y Galeano), serán los guías de la problematización que realiza el autor sobre la propuesta de Freire para una educación dialógica y liberadora.

Por su parte, el segundo capítulo de este volumen se titula $L a$ Filosofia y su enseñanza en Costa Rica. El currículo oculto en la pedagogía de la liberación intercultural: referentes teórico-prácticos.

En el artículo denominado La interculturalidad en la formación de profesores de enseñanza de la filosofía: un encuentro con la comunidad de Kcha'bli, Juan Gómez expone un repaso histórico de cómo surgieron las condiciones para entablar las relaciones que hoy en día se 
manejan entre académicos de la Universidad Nacional y la comunidad indígena Bribri de Kcha'bli (Kachabri en español) en Talamanca.

El autor plantea la necesidad de una perspectiva intercultural para establecer condiciones de horizontalidad e igualdad entre comunidades, asumiendo como punto de partida el diálogo y los consensos entre interlocutores. Históricamente, la separación disciplinar de los saberes se establece mediante una jerarquía del conocimiento científico por sobre los demás conocimientos (social, cultural, religioso, etc.). Siendo así, y desde la perspectiva de la ideología hegemónica neoliberal en nuestro contexto, los saberes indígenas no están legitimados para entrar en diálogo con los saberes científicos. Sin embargo, en la contemporaneidad se han señalado diversas similitudes entre las perspectivas cosmogónicas indígenas (descartadas desde el positivismo científico, como sugiere Gómez) y la cosmología (legitimada por el saber científico). En estas circunstancias, la pedagogía intercultural puede brindar las herramientas necesarias para vislumbrar y desnaturalizar estos esencialismos culturales, y así generar diálogos que conduzcan hacia una buena convivencia.

La importancia de este encuentro cultural entre la comunidad indígena de Kcha'bli y el estudiantado de Enseñanza de la Filosofía, concluye Gómez, es la posibilidad de experimentar otras relaciones con el mundo a partir de otros enfoques filosóficos, y a la vez cuestionar los axiomas bajo los cuales se asume esta instrucción, pues el claustro universitario tiende a entender la filosofía solamente como pensamiento occidental.

Seguidamente, en Educación, comunidad y liberación. Comentarios a partir del pensamiento pedagógico de Paulo Freire y Alejandro Cerletti: aportes a la enseñanza de la filosofía, también de Juan Gómez, se hace especial hincapié en el papel de la totalidad social comunitaria para plantear una pedagogía que, además de ser crítica y rigurosa con su contexto y sus condiciones, sea pertinente, significativa y transformadora de la realidad. Bajo esta perspectiva, tanto Freire como Cerletti brindan valiosas herramientas de análisis para problematizar la realidad a partir de lo emergente en nuestra relación con los demás.

Vislumbrar la práctica educativa dentro de un contexto holístico permite, como señala el autor, la revisión de categorías bajo las cuales construimos nuestra realidad, como la ética de la vida, de la comunidad y de la liberación. Y es que el hecho educativo no se limita (ni se puede limitar) a un sistema, sino que involucra toda la experiencia 
de vida del individuo. Las prácticas de enseñanza-aprendizaje son constantes en cualquier escenario, más allá de las aulas o los espacios institucionalizados.

Para lograr lo anterior, desde Freire, debemos primero vislumbrar la opresión que le genera el sistema educativo al sujeto para así proponer resistencias y alternativas. Estas alternativas, a su vez, tienen más sentido si se acogen directamente desde las voces de los y las oprimidas. El análisis de lo aleatorio, como propone Cerletti, implica escuchar atentamente esas voces y responder a ellas desde nuestra práctica educativa. Esto lleva a la necesidad de una educación contextualizada que problematice y, finalmente, rompa los esquemas homogeneizantes del sistema tradicional.

Por otra parte, Omar Ureña y Adrián Mata exponen en el texto titulado ¿Qué sabemos que se enseña en filosofía?: el currículo oculto de UNA formación filosófica las diferencias tangibles entre el currículo oficial y el currículo oculto encontradas en el Plan de Estudios del Bachillerato en Filosofía de la Universidad Nacional. En primera instancia, mediante un abordaje histórico, los autores discurren sobre las condiciones que mediaron la institucionalización de la Filosofía en Costa Rica, y el surgimiento en la UNA de aquel entonces, Departamento de Filosofía.

Desde su institucionalización en la UNA, la filosofía fue contemplada como una disciplina accesoria que estaba, literalmente, al servicio de otras disciplinas. El Departamento ofertaba diversos cursos de servicio a otras unidades académicas, hasta que se empezaron a ofertar posgrados con el surgimiento del Doctorado en Pensamiento Latinoamericano, y la propuesta de instauración de un Bachillerato en Filosofía que difiriera del ya existente en la UCR.

Como se mencionó anteriormente, este bachillerato surgió con un requisito que ninguna otra carrera tenía en ese entonces, y era la necesidad de aportar 40 créditos de una disciplina complementaria; es decir, de otra carrera universitaria. El problema es que, en los distintos documentos que sustentan el surgimiento del bachillerato y el plan de estudios, no hay claridad sobre los cursos o créditos que pueden convalidarse dentro de este apartado. Siendo así, el currículo oficial plantea que este requisito puede solventarse con la presentación de créditos de cualquier otra carrera (área no filosófica); pero en la práctica se dio la no validación de la pedagogía como una disciplina o "eje de metareflexión". 
Esta situación desembocó en una discusión entre varias posturas respecto al contenido del plan de estudios; y se demostró finalmente, que muchas de las pretensiones iniciales del currículo oficial del Bachillerato en Filosofía no se cumplen en la práctica. La idea de los 40 créditos tiene como punto de partida que, en la sociedad contemporánea, la filosofía no constituye una disciplina con la cual el estudiantado se pueda enfrentar a las condiciones del mercado laboral. Es por esta razón que se establece un enfoque filosófico interdisciplinar, según el cual el bachillerato brinda herramientas de análisis y diálogo al estudiantado para que generen relaciones entre la filosofía y sus otras disciplinas. Sin embargo, los autores señalan que esto no se da en el bachillerato, pues las posibilidades de diálogo son prácticamente nulas al finalizar el plan de estudios, teniendo en cuenta que este es eurocéntrico, realiza una exclusión a la mujer en sus contenidos y su estructura actual es contradictoria a los fines que representa.

Finalmente, Juan Gómez y Omar Ureña, en La enseñanza de la filosofia desde nuestra realidad latinoamericana: el caso de la Universidad Nacional de Costa Rica, retomando la separación disciplinar producto de la modernidad europea, plantean que se debe superar la noción de que la Filosofía responde a una racionalidad instrumental y reflexionar, filosóficamente hablando, nuestra realidad y contexto latinoamericano, tomando en cuenta a todos sus participantes desde un planteamiento que nos asuma, de entrada, como iguales en términos sociales.

Adoptando un posicionamiento que reconozca los saberes estéticos, más allá del cientificismo positivista, se puede generar un diálogo experiencial que tome en cuenta la realidad de cada sujeto y de su comunidad. Sobre esto, ¿qué acciones se ejecutan en la Universidad Nacional para responder a esta dialogicidad?

Esperamos entonces que el presente texto constituya un aporte para ustedes, que incite a la reflexión de nuestra práctica docente.

Juan Gómez Torres Adrián Mata Calderón

Omar Ureña Soto 